\title{
Applications of drones in emerging economies: a case study of Malaysia
}

\begin{abstract}
Drones or unmanned aerial vehicles (UAVs) are becoming increasingly popular for commercial and noncommercial uses - especially in fields of environment, surveillance, aerial photography, digital communications, search and rescue operations and military. Drones are in fact low cost aerial robots, that require little preparation and infrastructure and can be equipped with any number of sensors or cameras making them ideal for monitoring the environment. Environmental monitoring plays a major role in analyzing climate and management impacts on natural, agricultural systems, assessing, forecasting and even preventing natural disasters and enhancing hydrological cycle. Monitoring and data collection systems are based upon a combination of ground-based measurements and remote sensing sensors observations. These data however have spatiotemporal constraints. Drones offer an opportunity to bridge the existing gap between field observations and remote sensing by providing high spatial detail over relatively large areas in a cost-effective way. Drones have become popular in several developed countries in recent years. However, the use of drones is still in the infancy stage of development at developing countries such as Malaysia. This paper attempts to review the development of drone applications in Malaysia in order to identify future directions, applications, developments and challenges. We summarize that, to leverage the full potential of drones approaches in Malaysia, measurement protocols, retrieval algorithms, and processing and evaluation techniques need to be harmonized to ensure the sustainability and resiliency of the implementation.
\end{abstract}

Keyword: Component; Drone; Sector; Malaysia 